\title{
Unpaired Data
}

National Cancer Institute

\section{Source}

National Cancer Institute. Unpaired Data. NCI Thesaurus. Code C81020.

Data obtained from observations where there is a no link between an observation in one set of measurements and a particular observation in the other set of measurements. 